\title{
Energy Monitoring Platform for Smart Grid Applications
}

\author{
Juraj Brenkuš, Viera Stopjaková and Miroslav Slávik \\ Institute of Electronics and Photonics \\ Slovak University of Technology \\ Ilkovičova 3, 81219 Bratislava, Slovakia \\ Email: juraj.brenkus@stuba.sk
}

\begin{abstract}
The traditional power grid concept is suffering from lack of energy flow control mechanism. This is due to absence of distributed energy generation and storage nodes along the grid. With introduction of the Smart grid concept, this topic is addressed. However, such concept requires information on actual energy consumption that are distributed among the Smart grid flow control nodes in a secure way. These information should be as detailed as possible. This paper presents a consumption monitoring platform that is suitable for household and office building installations. The consumption data are gathered via wireless networks, while security is addressed on the application layer.

Index Terms-Smart grid, Energy monitoring, Wireless communication
\end{abstract}

\section{INTRODUCTION}

The power grid, as we know it today, is an unidirectional system, where the energy flow is not controlled and only limited types of power sources exist along the grid. This poses severe issues with energy demand fluctuations and distribution. In such cases, a local increase in energy demand can affect a fairly large portion of the grid. One of the solutions to this issue employs the introduction of local energy generation and storage facilities into the grid. These generation facilities are often based on renewable energy sources. With the local energy storage facilities, distributed along the grid, a more stable and balanced energy flow can be provided on the global level. However, to provide control over the energy flow in the grid, communication infrastructure has to be introduced that will ensure proper data exchange within the grid and its components, while data security needs to be addressed properly [1], [2], [3].

Energy flow monitoring is the essential property of the smart grid infrastructure. Grid balancing and energy flow optimization can be based on the data gathered from monitoring devices deployed on different levels along the smart grid: from generation, distribution and storage, down to the end user level. From a global perspective, these data can be used to create predictions based on typical user behavior. From the end user point of view, creation of the optimized consumption scenarios could be an example of energy flow monitoring usage. In this paper, we propose an energy consumption monitoring platform intended for data gathering and device reconfiguration on the lower level of the smart grid, i.e. households and office buildings.

\section{PROPOSED ENERGY CONSUMPTION MONITORING SYSTEM}

Leveraging on the requirements emerging from the foreseen future smart grid operation, we have proposed an energy monitoring platform that is based on well-known communication standards to ease its integration, while due attention is paid to the security topic on the application layer. The platform is suitable for deployments in households, office buildings and small-scale industrial applications, where the energy consumption data are required to be monitored as far as the wall outlet level. Beyond this point, only intelligent appliances can provide additional information, e.g. on energy consumption prediction based on the actual state. Such information could be very helpful in creation of energy demand forecasts.

The proposed platform consists of the following main components:

- Dual interface sensor node (Metering socket)

- Long range communication gateway

- Application server

- Mobile device (optional)

The model of the proposed metering platform is depicted in Fig. 1 in more details. The basic idea is to have as little installation overhead as possible. Therefore, we have opted for communication protocols that have decent market penetration and are power efficient, while still fulfilling the requirements on M2M communication within the Smart grid. The sensor nodes are equipped with two communication interfaces:

- short-range, using Bluetooth 5 technology

- long-range, based on LoRaWAN ${ }^{\mathrm{TM}}$ technology

The short-range communication interface is normally used to initialize and configure the device, or to view live measured data from a selected device. However, this functionality is not critical for the operation of the monitoring system, but improves the user experience and eases the (re-)configuration of the network in a more secure way. This interface can also serve as a second communication channel with the application server. Moreover, if the density of the sensor nodes is sufficiently high, a mesh network is created using these interfaces. The mesh configuration allows the users to connect to a large network at any point and communicate with arbitrary (even very remote) devices within the network. This is also possible with Bluetooth Low Energy devices not supporting Bluetooth 5 technology. 


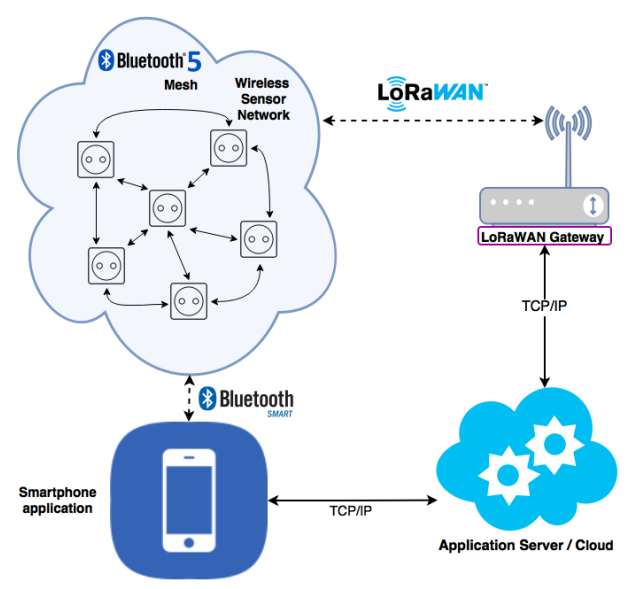

Fig. 1. High level model of the proposed platform.

Bluetooth 5 is an IoT extension of the previous Bluetooth Low Energy with the improved range, data throughput and capability of mesh network. The Bluetooth is built on two core profiles Generic Access Profile (GAP) and Generic Attribute Profile (GATT). The GAP is handling Bluetooth broadcasting and connecting roles. The GATT defines a hierarchical data structure that is exposed to connected Bluetooth devices [4].

The data measured by the sensor nodes is sent to the application server via the long-range communication interface periodically, in predefined time intervals. This interface communicates with the application server via gateways that transparently forward the encrypted data between the sensor nodes and the server. If several gateways are in the reach of the sensor interface, all of them forward the data to the application server, which takes care of the redundant data packets. In fact, the long-range communication gateway is the only installation overhead that is, beside the sensor nodes, required for the platform setup. However, there are also gateways that are publicly available and can be also used as a part of the infrastructure. Even in such configuration, the data security is not compromised since is addressed on the application layer.

LoRaWAN is a low power and long-range network designed for IoT sensors and actuators. The network can be optimized with parameter named spreading factor either for long range and low data rate, or for short range and high data rate. The LoRaWAN network in Europe can be deployed on free ISM $868 \mathrm{MHz}$ band, restricted only by the transmittion duty cycle. Typical network range is about $2-3 \mathrm{~km}$ in city around the gateway [5].

\section{DESCRIPTION OF METERING SOCKET}

The proposed Metering socket solution is embedding an energy consumption measurement system, longrange and short-range wireless communication interfaces and switching circuitry into a single compact device, which can fit into a standard wall outlet installation box. The metering system is able to measure voltages and currents up to $230 \mathrm{~V}$ and $16 \mathrm{~A}$,
TABLE I

COMPARISON OF EXISTING SOLUTIONS

\begin{tabular}{c|c|c|c} 
Product & $\begin{array}{c}\text { Communication } \\
\text { interface }\end{array}$ & $\begin{array}{c}\text { Rated current } \\
{[\mathrm{A}]}\end{array}$ & $\begin{array}{c}\text { Consumption } \\
{[\mathrm{W}]}\end{array}$ \\
\hline \hline$[6]$ & $868 \mathrm{MHz}$ & 16 & 0.6 \\
{$[7]$} & Zigbee & 10 & N/A \\
{$[8]$} & BT4 & 11 & N/A \\
{$[9]$} & Z-Wave & 11 & 1.6 \\
This work & BT5 + LoRa & 16 & 0.7
\end{tabular}

respectively. The metering system is also capable of switching loads drawing current up to $16 \mathrm{~A}$, and provides an integrated residual-current circuit breaker solution. Such a solution is to best of our knowledge not available on the market in such a form factor.

\section{A. Existing solutions}

In Tab. I we compare the solutions available on the market in our region. All of these solution are capable of power consumption measurement and load switching. It is worth noting that none of the solutions is available as a module that could be built into a wall outlet. Moreover, several solutions deploy communication protocols that require dedicated (usually expensive) data acquisition units. The values for the consumption represent the maximum possible power consumed by the metering device according to their specifications.

\section{B. Hardware}

Main hardware components of the proposed system are:

- Micro-controller unit (MCU) with integrated short-range communication interface

- Hardware secure element

- Measurement front-end

- Long-range communication interface

- AC/DC power converter

- Residual-current circuit breaker (RCCB)

A more detailed visualization of the Metering socket's building blocks is given in Fig. 2 .

The selected ARM-based MCU, which controls the overall system functionality, is equipped with a Bluetooth 5 radio transceiver controlled by Bluetooth qualified software protocol stack. For specific usecases, the integrated short-range NFC tag interface can also be utilized for authentication purposes (e.g. OOB for Bluetooth). Main features of the MCU include:

- 32-bit ARM Cortex-M4F Processor (58 $\mu A / M H z$ CPU efficiency)

- $512 \mathrm{kB}$ flash + $64 \mathrm{kB}$ RAM

- Configurable I/O mapping for analog and digital $\mathrm{I} / \mathrm{O}$

- Up to $+4 \mathrm{dBm}$ output power

- $-96 \mathrm{dBm}$ sensitivity (Bluetooth low energy)

A more detailed description of the MCU parameters is given in [10].

The main role of the measurement front-end is to provide sensing, analog-to-digital data conversion, and 


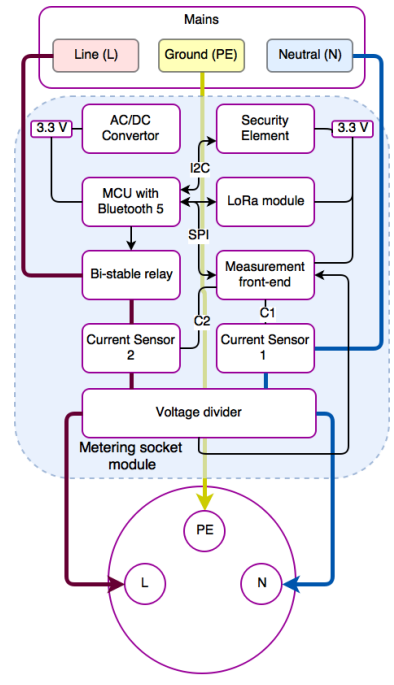

Fig. 2. Block diagram of the proposed Metering socket.

data preprocessing for the MCU. The STPM33 measurement front-end by $S T$ Microelectronics provides three measurement channels (two for current and one for voltage measurement) [11]. It also integrates a DSP, and therefore, is capable of providing the large scale of parameter measurements on a single phase line, e.g. energy/power (active, fundamental, reactive, apparent), phase shift, frequency, voltage/current (RMS and instantaneous), etc. The RMS values of the voltage and both currents are calculated based on the well known equations 1 and 2 over the period of $\mathrm{T}=200 \mathrm{~ms}$.

$$
\begin{aligned}
V_{R M S} & =\sqrt{\frac{1}{T} \int_{t_{0}}^{t_{0}+T} v(t)^{2} d t} \\
I_{R M S} & =\sqrt{\frac{1}{T} \int_{t_{0}}^{t_{0}+T} i(t)^{2} d t}
\end{aligned}
$$

The dynamic range on the active power measurement is up to $10000: 1$, while the measurement error is kept below $0.5 \%$. The active power is calculated for the whole frequency range of $4 \mathrm{~Hz}-3.6 \mathrm{kHz}$, and for the fundamental frequency in the range of $45-65$ $\mathrm{Hz}$ separately. The measured values are stored in the register set that can be accesed via the SPI interface. The communication with the host MCU is performed via SPI with a 2 byte Cyclic Redundancy Check (CRC) protection to ensure correct data transfer.

For long-range communication, a LoRa transceiver based on Semtech's SX1276 is used [12]. This interface uses $868 \mathrm{MHz}$ ISM band. The transceiver is controlled by a communication protocol stack running in the main MCU. This interface is capable of data transfer over the distance of several kilometers.

The RCCB is implemented as a bi-stable relay, controlled directly from the measurement front-end. Huge advantage of the bi-stable implementation is no static power consumption, as the control impulse is needed only when the relay state has to be switched. The control impulse generation is governed by the current difference measured on the two current sensing lines.

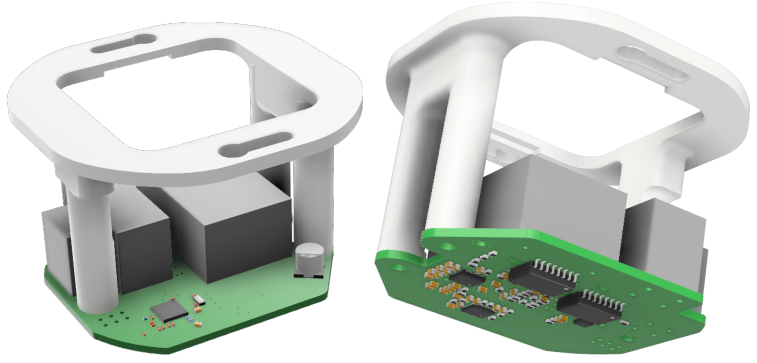

Fig. 3. Visuals of the proposed Metering socket module.

Since the Smart grid represents a critical infrastructure, a dedicated HW secure element (SE) has been deployed to ensure the highest possible level of data security and device authenticity within the network. SE is a security hardened HW platform from NXP used to store keys and provide cryptographic operations with these keys. The authentication keys are transferred to the SE upon the system installation from a trusted source. After this initialization of the SE, the keys will never leave it, thus they can not be compromised. Another use-case is that the keys are generated directly in the SE, and only the public key is made available for the devices that need to communicate with the Metering socket. The SE is connected to the host MCU via $\mathrm{I}^{2} \mathrm{C}$ bus.

Visual of the proposed HW solution is shown in Fig. 3. This picture shows the PCB of the metering socket including the fixture to position the PCB within a socket.

\section{Software}

The whole monitoring system is controlled by nRF52832 with ARM Cortex-M4F Processor that provides:

- Running Bluetooth 5 and LoRaWAN ${ }^{\mathrm{TM}}$ protocol stack

- Controlling the measurement front-end (configuration, measured data readout)

- Controlling switching circuitry, triggering RCCB

- Data management (storing, sharing, encryption)

- Communication with secure element

A brand new Metering socket has to be connected to a smartphone application for the initial configuration. After the first setup both long-range and short-range communication interfaces are enabled, and the measurement can start. Besides the standard Bluetooth services, the following custom services are implemented in our measurement hardware:

- Device status

- Actual data

- Historical data

Device status service includes characteristics for device configuration, such as LoRaWAN credentials or bi-stable relay state and for device events like RCCB trigger.

Actual data service is meant to transfer real-time measurement data via Bluetooth to a mobile application. Data measurement and data transfer are started 
TABLE II

POWER CONSUMPTION EVALUATION

\begin{tabular}{c|c|c} 
Scenario & $\begin{array}{c}\text { DC domain } \\
\text { consumption [mW] }\end{array}$ & $\begin{array}{c}\text { AC domain } \\
\text { consumption }[\mathrm{mW}]\end{array}$ \\
\hline \hline Idle & 14 & 82 \\
Meas & 57 & 160 \\
Meas + BT5 & 84 & 205 \\
Meas + LoRa & 73 & 163
\end{tabular}

with enabling notification on the data transfer characteristic. Furthermore, the service consists of characteristic which defines the notification interval and a characteristic for selecting data to be reported.

Historical data service is similar to actual data service but this service is exposing the already measured data stored in the MCU's flash memory. Measurement period and data to be transferred are defined by separate characteristics analogous to the actual data service implementation. Stored data can be transferred via read-only data transfer characteristic.

The LoRaWAN interface is used for harvesting historical measurement data and for triggering the bistable relay. Actual measured data cannot be send via LoRaWAN due to transmittion duty cycle regulations and the consequent delay in the data reporting.

The Metering socket configuration as well as the measured data are stored in dedicated pages of the MCU's internal flash memory, where each record is extended by a CRC to ensure data validity. This data are encrypted using the SE before transmission, so only the target device (Application server, mobile phone) can decrypt them. Before any data transmission, authentication of the transmitting device is requested by the receiving device. For this action, the keys stored in the SE are used and temporary session keys are generated for a given time period.

\section{Power consumption}

Since power efficiency of the measurement system is one of the main design goals, power consumption of the selected components was elaborated thoroughly. MCU as the central device of the Metering socket wakes up only when requested by the communication protocol stacks, SE or data read-out needs to be performed from the measurement front-end. All the peripherals that are not needed at the given time, are forced to a sleep mode to save power. Power efficiency of the proposed solution was elaborated on development boards, while different performance scenarios were simulated. The consumption was evaluated on both the 3.3 V DC supply line, after conversion from AC voltage of $230 \mathrm{~V}$ using a switch mode power supply module, and from the supply line as well. The results of these measurements are summarized in Tab. II. The values shown here represent the average consumption for the given scenarios.

\section{CONCLUSIONS}

A wireless energy metering platform is presented in this paper. The communication is based on two standard protocols, one for short- and the other one for long-range communication. The data gathered on a wall outlet level are sent to a remote location (e.g. cloud, application server, etc.), where they are stored, analyzed and can be used to visualize historical consumption data, create demand predictions and to control the energy flow in the grid.

The only solution feasible to large-scale wireless energy metering deployments within the smart grid is the one that uses the frequency spectrum and the energy from the power supply efficiently, so the foreseen benefits in terms of grid stability and energy flow prediction will be available at only a small cost increase in the overall consumption, while the communication band utilization will be kept low. The solution presented in this paper kept these requirements as the foremost design goals.

Significant advantage of a wireless network is the ease of extension by new nodes. Once the appliances would become intelligent in the sense that they are able to send information via a standard powerefficient communication interface with mesh support, e.g. Bluetooth 5, they could be easily integrated into an existing smart home network without the need of an additional communication infrastructure installation (e.g. dedicated gateway, repeater, etc.). Such a concept would create a valuable source of information for energy demand prediction and scheduling.

\section{ACKNOWLEDGMENT}

This work was supported in part by the Research and Development Agency under grant APVV-15-0254, ECSEL JU under project CONNECT (737434) and the Slovak Republic under grant VEGA 1/0905/17.

\section{REFERENCES}

[1] G. F. D. G. Apolinario, M. J. S. Dizon, V. O. Gaspar and G. L. T. Obligado, "Design of smart monitoring and communication system for NCR power grid," 2017IEEE 9th International Conference on Humanoid, Nanotechnology, Information Technology, Communication and Control, Environment and Management (HNICEM), Manila, Philippines, 2017, pp. 1-6.

[2] S. Kaur and S. Kaur, "Comparative analysis of lightweight cryptography algorithms for smart grids," 2017 4th International Conference on Signal Processing, Computing and Control (ISPCC), solan, India, 2017, pp. 564-567.

[3] E. U. Haq, H. Xu, L. Pan and M. I. Khattak, "Smart Grid Security: Threats and Solutions," 2017 13th International Conference on Semantics, Knowledge and Grids (SKG), Beijing, China, 2017, pp. 188-193.

[4] Available online: https://www.bluetooth.com/specifications/gatt/genericattributes-overview

[5] Available online: https://www.lora-alliance.org/what-is-lora

[6] Available online: http://www.revogi.com/smart-plug-eu/

[7] Available online: http://www.centralite.com/downloads/DataSheet3SeriesSmartOutletTypeE.pdf

[8] Available online: https://www.elgato.com/en/eve/eve-energy

[9] Available online:

[10] Available online: http://infocenter.nordicsemi.com/pdf/nRF52832_PS_ v1.4.pdf

[11] Available online: http://www.st.com/content/st_com/en/products/dataconverters/metering-ics/stpm33.html

[12] Available online: https://www.semtech.com/products/wirelessrf/lora-transceivers/SX1276 